\title{
Qualitative Recognition of Typical Loads in Low-Speed Rotor System
}

\author{
Kun Zhang ${ }^{1,2}$ and Zhaojian Yang ${ }^{1,2}$ \\ ${ }^{1}$ College of Mechanical Engineering, Taiyuan University of Technology, Taiyuan 030024, China \\ ${ }^{2}$ Shanxi Key Laboratory of Fully Mechanized Coal Mining Equipment, Taiyuan 030024, China \\ Correspondence should be addressed to Zhaojian Yang; yangzhaojian@tyut.edu.cn
}

Received 15 May 2017; Revised 11 August 2017; Accepted 1 October 2017; Published 26 October 2017

Academic Editor: Yuri Vladimirovich Mikhlin

Copyright (c) 2017 Kun Zhang and Zhaojian Yang. This is an open access article distributed under the Creative Commons Attribution License, which permits unrestricted use, distribution, and reproduction in any medium, provided the original work is properly cited.

\begin{abstract}
While the load variations within the low speed rotor systems affect the operating conditions and mechanical properties, they may also provide information on machine faults. Therefore, load recognition is of great significance in operational monitoring for detecting early warning signs of failure and diagnosing faults. In this paper, five types of typical loads in a low-speed rotor system are qualitatively analyzed. Moreover, a method is presented based on the vibration signals from a low-speed rotor system using the ensemble empirical mode decomposition (EEMD), energy feature extraction, and backpropagation neural network (BPNN). A low-speed rotor test bench was designed and manufactured for load recognition and an experiment was set up based on certain load characteristics. Loading tests for five representative categories were conducted and various vibration signals were collected simultaneously. The EEMD was shown to eliminate the mode mixing seen in traditional EMD, which resulted in a clear decomposition of the signal. Finally, the characteristics were imported into a BPNN after energy feature extraction, and the different types of load were accurately recognized. Comparing the experimental results to existing data, a total recognition rate of $92.38 \%$ was achieved, demonstrating that the proposed method is both reliable and efficient.
\end{abstract}

\section{Introduction}

Modernization of machines has resulted in rotating parts that are often subjected to complex and variable loads during operation, which could potentially lead to costly production losses and catastrophic failure [1]. While the load variations within the low-speed rotor systems affect the operating conditions and mechanical properties, they may also provide information on machine faults. Variable loads experienced by rotating machines include large impact loads while reversing or stopping, steady loads during smooth operation, and harmonic loads in the case of misalignment or unbalanced failures. Other examples include reciprocating rolling plates such as a roller system in a rolling mill under transient load and spindle rotor systems in winding hoists required to withstand linear loads due to weight changes within the hoisting rope. During these processes, load changes can directly affect normal operation conditions and by recognizing the load type it is possible to qualitatively determine the running status of the equipment. Load recognition may also be of great significance in operational monitoring for detecting early warning signs of failure and diagnosing faults. However, it is not a trivial task to directly measure continually changing load values due to varying conditions. Thus, in many engineering systems it is difficult to ascertain the operating loads of a rotor system. In addition, load recognition is a key factor in dynamic structure design and postphase state monitoring. Therefore, load recognition within a low-speed rotor system is a complex and systemic problem and should be determined by appropriate load recognition methods [2].

The goal of load recognition is to identify the type of dynamic load in a system. The methods used for load recognition are based on known characteristics of the system as well as actual measurements of the dynamic response. Load recognition is considered the second inverse problem of the dynamic response of a system [3] and focuses on nonstationary characteristics and inversion of the signal. Several research groups have actively pursued research in load 
recognition. Dobson and Rider developed a method for the indirect calculation of excitation forces using measured structural response data [4]. Inoue et al. performed an inverse analysis by indirect measurement of an impact force [5]. Moreover, Jankowski considered the off-line recognition of spatial and temporal characteristics of a dynamic load [6]. Klinkov and Fritzen presented an updated overview of available time domain load reconstruction methods [7], while Czop et al. proposed an approach for the detection of rail truck irregularities based on measurements of the bearing box acceleration [8]. Finally, Yu and Chan reviewed load recognition techniques for moving loads on bridges [9].

Frequency and time domain methods are traditionally used in load recognition approaches $[10,11]$. The frequency domain recognition method has been comprehensively reviewed in [11]. The basic idea of frequency domain method is to establish the input and output frequency function of the system in the frequency domain. According to the vibration theory, the frequency response function is a linear operator of which the inverse operation is easy to handle. Therefore, the problem of determining loads by the responses can be transformed into solving linear equations [12]. The model inverse operation of frequency domain method is easy to process and its dynamic calibration is simple. However, in aspect of recognition accuracy, frequency response model function can cause an instable recognition and a decreased accuracy due to the matrix morbid. In terms of application scope, the frequency domain method is applicable to recognize stable loads $[13,14]$. Time domain method was a method that emerged only in the 1980s. It started late compared with load identification method in frequency domain [15]. Its main idea is based on the relationship between load and its response. After motion equations conducted modal transformation, noncoupled dynamical equations can be obtained by the dynamic equation decoupling. Finally, time history of dynamic load can be solved. However, the sensitivities of initial value and boundary conditions make an unsatisfactory recognition accuracy. Meanwhile, modal parameters and the high mode truncation can also cause an error accumulation. In terms of computation amount, the discrete convolution relation of model function is more complex, resulting in a heavy workload $[5,16,17]$. With the development of computer technology, new algorithms have been introduced into the field of load recognition [18-22]. Neural networks are being developed and have excellent potential in this area $[3,23]$. Neural networks are applicable to all types of transient load and have high recognition accuracy [24].

Modern methods of load recognition are rarely used in the rotor systems of rotating machinery. We have studied the load recognition of high-speed rotor system and obtained good recognition results [25]. Meanwhile, we found that some important industrial and mining equipment such as large-scale cranes, hoisting systems, and heading machines are required to run continuously at low speeds. In view of the low-speed working conditions which are different from the high-speed conditions we previously studied, we are also interested in the load identification of low-speed rotor system. Furthermore, load-type recognition of lowspeed rotors has rarely been reported. Therefore, the paper chooses this as a pointcut, conducting the relevant research and discussion. For this purpose, a low-speed rotor system test bench was specially designed and constructed to verify the feasibility of its load identification method. The proposed recognition method can also be used online by linking the recognition function with a signal-monitoring interface allowing for real-time monitoring of equipment loads during operation.

\section{Theories and Methods}

The recognition method for recognizing the load category of a low-speed rotor system can be shown as a flowchart (Figure 1). First, the vibration signals triggered by the load are detected as input. Following this, the next steps include signal preprocessing, energy feature extraction, and recognition of the load category. Finally, the results of the load recognition are output.

The EEMD method is utilized as the preprocessor for vibration signals and the energy calculations and BPNN is then applied as the load category recognition process.

2.1. Signal Preprocessing. EEMD is a noise-assisted data analysis (NADA) method that works by adding white noise in order to improve the signal analysis and can therefore eliminate mode-mixing phenomena which occur using the traditional EMD method [26]. Mode-mixing phenomenon can occur during the process of the EMD decomposition, whereby a signal within the same frequency range is decomposed into different IMFs or an IMF including multiple signals of different frequencies, in particular when the original signals contain interstitial or pulse components. The EEMD method improves this problem by superimposing the original signal Gaussian white noise and repeatedly decomposing the signal with EMD (Figure 2). As the statistical property of Gaussian white noise is uniformly distributed, signal components of different scales are automatically distributed to appropriate reference scales. Accordingly, the degree of mode aliasing in each IMF component is reduced [27].

The EEMD method is composed of the following steps:

(1) A sequence of Gaussian white noise $n_{j}(t)$ is added to the original signal $x(t)$, which is expressed as

$$
\tilde{x}(t)=x(t)+n_{j}(t) .
$$

Standard deviation of white noise $\sigma_{n}$ can be determined according to the following steps:

Step 1: calculate amplitude Std Dev of the original signal $\sigma_{0}$.

Step 2: original signal is conducted with high-pass filtering. Amplitude Std Dev of the high-frequency component $\sigma_{h}$ can be calculated.

Step 3: according to the probability distribution in normal function, its probabilistic relation is $P(\mu-$ $3 \sigma<x<\mu+3 \sigma)=99.73 \%$. When $\sigma_{n}=\sigma_{h} / 3$, the probability of white noise $n(t)$ 's amplitude distribution in $-3 \sigma_{n}<n(t)<3 \sigma_{n}$ is $99.73 \%$. $99.73 \%$ of 


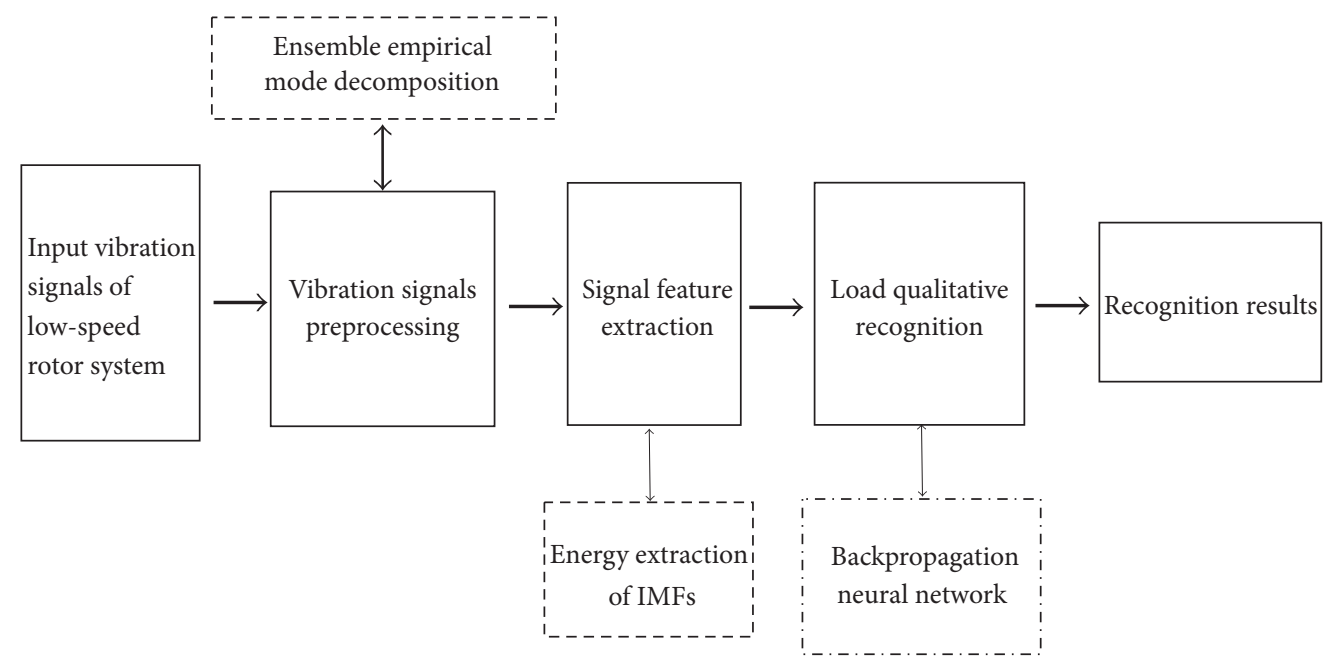

FIGURE 1: Flowchart of the main steps involved in the load recognition method for the load categories of a low-speed rotor system.

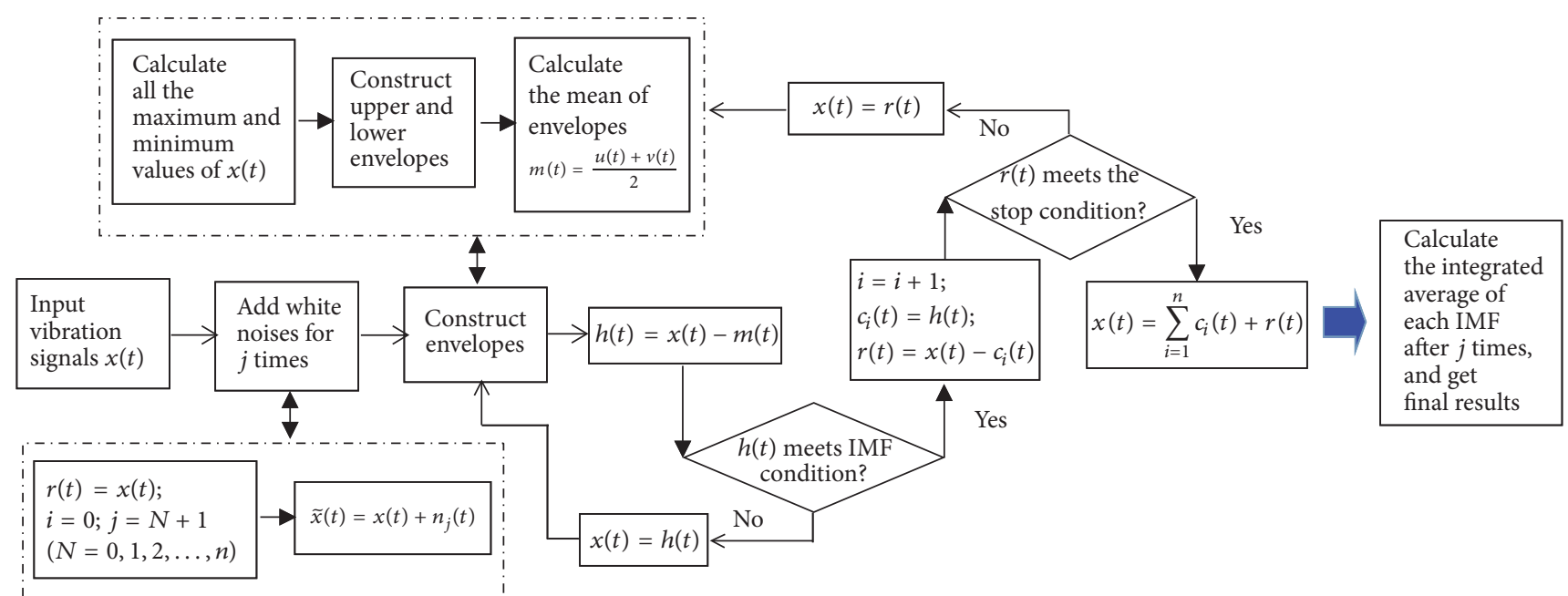

FIGURE 2: Flowchart of the EEMD process.

the amplitude absolute value for white noise discrete points is less than $\sigma_{h}$. At this time, the added white noises do not affect the extreme point distribution of high-frequency signal [28].

Step 4: the amplitude Std Dev of white noise can be obtained as

$$
0<\sigma_{n}<\frac{\sigma_{h}}{3}
$$

(2) Maximum and minimum points of the signal $\widetilde{x}(t)$ are calculated. Next, the upper and lower envelopes of the signal are constructed and the average signal $m(t)$ is calculated.

(3) The original data is subtracted from the average signal $m(t)$ to obtain $h(t)$ as

$$
h(t)=x(t)-m(t) .
$$

Upon determining whether $h(t)$ satisfies two IMFconditions using the whole data sequence, the number of extreme value points is set to be equal to the number of zero passing points. The maximum difference between the two numbers cannot be above a certain value and, at any point, the mean of the envelopes is zero, defined by the maximum and minimum local values of the signals. This is the highest frequency component of the signal sequence. Then, subtracting $c_{1}(t)$ from $x(t)$ gives a new data sequence $r(t)$ as follows:

$$
\begin{aligned}
i & =i+1 ; \\
c_{i}(t) & =h(t) ; \\
r(t) & =x(t)-c_{i}(t) .
\end{aligned}
$$

The stop condition of $r(t)$ needs to be checked. If the stop criteria are not met, $r_{1}(t)$ must be calculated again using the decomposition mentioned above. The process is repeated 
until the last data sequence $r_{n}(t)$ cannot be decomposed further.

$$
x(t)=\sum_{i=1}^{n} c_{i}(t)+r(t)
$$

(4) Repeat (1) for $N$ times. And a new sequence of Gaussian white noise $n_{j}(t)$ is added at each iteration.

(5) The IMF components are calculated using an integrated average processing, after being decomposed $\mathrm{N}$ times and the results are obtained.

2.2. Signal Feature Extraction. After each addition of white noise, the signal is processed by EEMD. Then the corresponding intrinsic mode function (IMF) can be obtained. After EEMD, each IMF shows a stationary signal with a set of characteristic scales. Different load features are represented by a characteristic energy change in each frequency band. After signal decomposition, the energy of each component and residual component is acquired. The residual component contains very little information and is therefore neglected. Due to the orthogonal decomposition, the total signal energy is equal to the IMF energy [29].

After EEMD processing of the signal, $n$ IMF components are selected which include the load information. The total energy $E_{i}$ of each IMF is calculated as

$$
E_{i}=\int_{-\infty}^{+\infty}\left|\mathrm{IMF}_{i}\right|^{2} d t, \quad(i=1,2, \ldots, n) .
$$

A feature vector $E$ is constructed from the total energy and given as $E=\left[E_{1}, E_{2}, \ldots, E_{n}\right]$. The obtained energy ratio can be defined as

$$
T_{i}=\frac{E_{i}}{\sum_{i=1}^{n} E_{i}} .
$$

When different loads are applied to a low-speed rotor, the displacement signals can be obtained. In each frequency band, the signal energy changes and contains abundant loading information. Thus, the signal feature vectors are extracted at this point from the IMF energy components.

2.3. Type Recognition Method. An artificial neural network (ANN) is a mathematical model simulating the biological neural network. While ANN is not required when sample data obeys a normal or particular distribution, it does have the ability to handle nonlinear problems and does not require determining whether there is a correlation among variables. A BPNN is a widely used type of multilayers forward neural network. The nonlinear relationship between the input and output variables is determined through training such that a relational model of the input and output can be established. The BPNN method has powerful computing ability and, theoretically, can approximately express any complex mapping. Furthermore, it is strongly applicable in the field of pattern recognition [25].

(1) Network Structure. The topological structure of the BPNN model is presented in Figure 3. The input values are

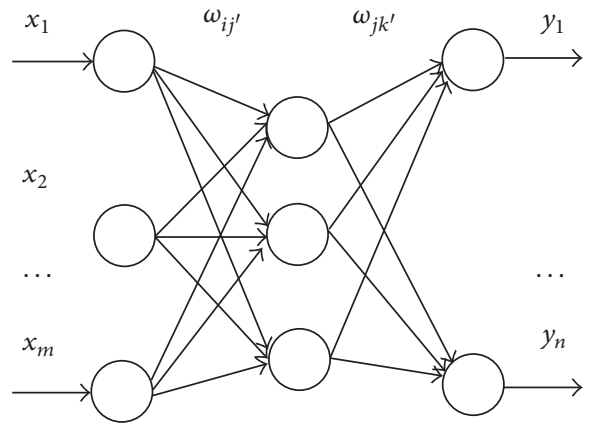

FIgURE 3: Topology of BPNN model.

$x_{1}, \ldots, x_{m}$ and the output values are $y_{1}, \ldots, y_{n}$, while $\omega_{i j}$ and $\omega_{j k}$ are the connection weights between the nerve cells in each layer.

The node numbers of the input layer $m$ and output layer $n$ are the number of input and output values in the network, respectively. The node number of the hidden layer $l$ can be determined by [19]

$$
l=\sqrt{m+n}+a,
$$

where $m$ is node number of the input layer, $n$ expresses node number of output layer, $l$ denotes node number of hidden layer, and $a$ is an adjustable integer constant between 1 and 10.

(2) Sample Generation. After signal processing and feature extraction, the original signals are divided into two parts: training samples and testing samples. The former are used to train the model and the latter are the samples that will be recognized. The sample data are the input of the network.

Here, the vibration signals, corresponding to the different loads applied to the rotor system, are collected. The signals, after EEMD and energy feature extraction, are input into the neural network. The data contains abundant load information.

\section{(3) Network Training}

(a) Sample Forward-Propagation. In forward-propagation process, the input is used to calculate the final output. The sample moves from the input layer to the hidden layer, then to the output layer. In each layer, neuron states only affect neurons in the next layer.

Output from the hidden layer is given by

$$
H_{j}=f\left(\sum_{i=1}^{n} \omega_{i j} x_{i}-a_{j}\right), \quad j=1,2, \ldots, l .
$$

Output from the output layer can be given by

$$
O_{k}=\sum_{j=1}^{l} H_{j} \omega_{j k}-b_{k}, \quad k=1,2, \ldots, m .
$$

(b) Error Backward-Propagation. Backward-propagation is a process whereby the weights and thresholds are modified by 
TABLE 1: Five types of coding load conditions.

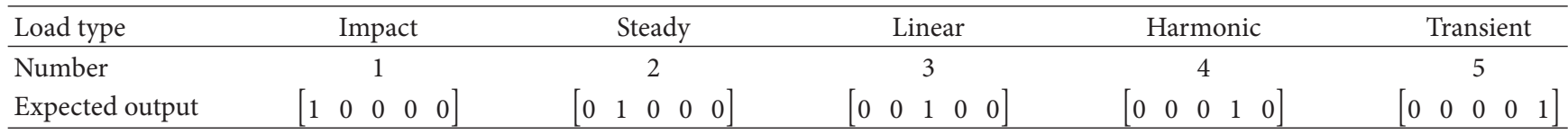

using the error. When the output layer does not obtain the desired result, the error backward-propagation begins. By continuously adjusting the cumulative values and threshold values the error is minimized. During the bidirectional propagating process, differences between the actual and desired output are recursively calculated and the differences are used to adjust the weights, continuing to learn until the error reaches the tolerance limit.

(4) Network Classification. After training, the BPNN has the ability to learn. The testing data can be classified using the network such that different types of load are recognized.

In this study, five load types were used as the network output. For the network coding of load types, an expected output of 1 indicates that the system is in the current load condition. For the five load types, classifications correspond to mathematical codes of the expected output, as shown in Table 1.

(5) Evaluation of Results. According to the classification accuracy, the final results were evaluated for network pattern recognition. A higher accuracy indicates a stronger ability to classify the load. The results of the load recognitions were then evaluated by comparing the predicted results with real load types.

The design of a suitable BP neural network, followed by training the network, will allow for the realization of a qualitative load recognition method for low-speed rotor systems. The process of combining the algorithm with the EEMD, energy extraction, and BP neural network is conducted before network training.

\section{Experiments}

3.1. Experimental Setup. The load recognition experiments were implemented on a specialized test bench, designed based on the purpose of this research. The system was programmed to control torsional loading, which was then applied to the rotor system. During loading, vibration signals were measured synchronously and the load-type recognition was conducted by analyzing the collected signals.

Five types and sizes of loads are ordinally applied to the rotor system through a brake to the system, as shown in Table 2. Each load is corresponding to a loading experiment for the rotor system. The first column expresses 5 types of loads in the experiments, namely, impact load, static load, linear load, harmonic load, and transient load. Among them, when applying impact load, it needs to keep $0.5 \mathrm{~s}$. For transient load, it needs to apply $3 \mathrm{~s}$. Other types of loads are conducted more than $10 \mathrm{~s}$. "Expression $(\mathrm{Nm})$ " presents mathematic expressions for loads. In the load expression table, $M$ is load torque, with unit of $\mathrm{Nm}$, and $t$ is time in s. The fifth line is taken as an example. The first item "Harmonic" means these load types are harmonic. The second to the fifth items show the sizes of harmonic loads applied in turn; namely, $M=200 \sin 4 \pi t+1500 / 200 \sin 20 \pi t+1500 /$ $100 \sin 4 \pi t+1550 / 100 \sin 20 \pi t+1550 \mathrm{Nm}$. Other lines are in the same way. For each loading, the corresponding vibration signals in rotor system are acquired synchronously. And realtime monitoring data are recorded.

There are some loads in five types to be recognized which are randomly selected, namely, impact loads $(1450 \mathrm{Nm}$, $1550 \mathrm{Nm}$, and $1650 \mathrm{Nm})$ for $0.5 \mathrm{~s}$, steady loads $(1450 \mathrm{Nm}$, $1550 \mathrm{Nm}$, and $1650 \mathrm{Nm})$, linear loads $(0.5 t+1600 \mathrm{Nm}, t+$ $1500 \mathrm{Nm}$, and $2 t+1550 \mathrm{Nm}$ ), harmonic loads (100 sin $20 \pi t+$ $1550 \mathrm{Nm}, 200 \sin 4 \pi t+1500 \mathrm{Nm}$, and $100 \sin 4 \pi t+1600 \mathrm{Nm})$, and transient loads ( $1450 \mathrm{Nm}, 1550 \mathrm{Nm}$, and $1650 \mathrm{Nm})$ for $3 \mathrm{~s}$.

During the experiment, loads were selected randomly. Both the training and testing signals were included in the vibration signal.

3.2. Test Platform Build. The low-speed rotor system, such as the mine hoist, is mainly composed of a drive device (e.g., motor), deceleration mechanism, and low-speed rotor. A test bench containing the main structures of the mine hoist was designed, manufactured, and debugged for more than one year. The main parts of the designed test bed include a mechanical unit within the low-speed rotor system, a torque actuator unit, and a signal test unit. Within the mechanical unit, the mechanical parts are driven by a three-phase asynchronous motor. The motor was designed with a power output of $37 \mathrm{~kW}$ and rated speed of $42 \mathrm{r} / \mathrm{min}$. The torque control unit is capable of creating different types and sizes of dynamic loads using a control card and magnetic powder brake. It should be noted that the brake must be monitored and cooled with water, and the continuous usage time cannot extend beyond $5 \mathrm{~min}$ to prevent overheating. Within the signal test unit, a displacement sensor was used to monitor the displacement vibration of the left bearing. The signal acquisition instrument "INV3060" has a sample frequency of $2048 \mathrm{~Hz}$.

A schematic showing the load recognition test bench is presented in Figure 4. The load recognition experiments were conducted on the test bench and various loading states were implemented. The displacement sensors were arranged within the rotor system such that the vibration signals corresponding to different loads were collected successfully. Finally, MATLAB (The MathWorks, Inc., Natick, MA, USA) was used for analysis and calculations.

\section{Results and Analysis}

4.1. Vibration Signal Enhanced by EEMD. Loading experiments for the five load types were conducted in the low-speed 


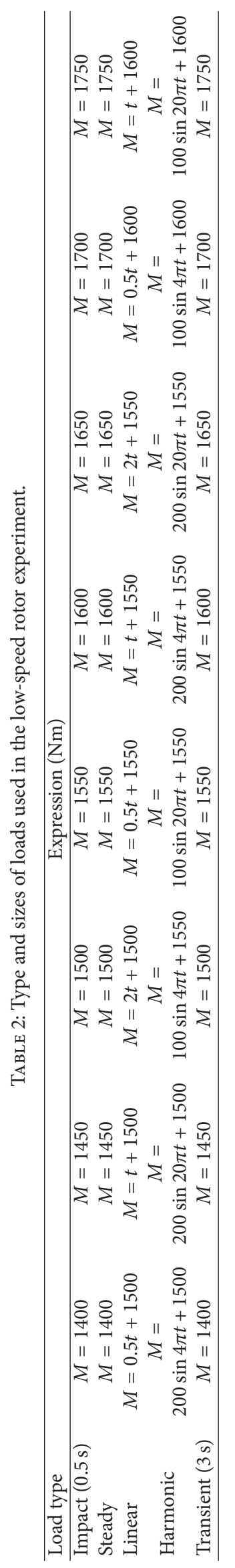




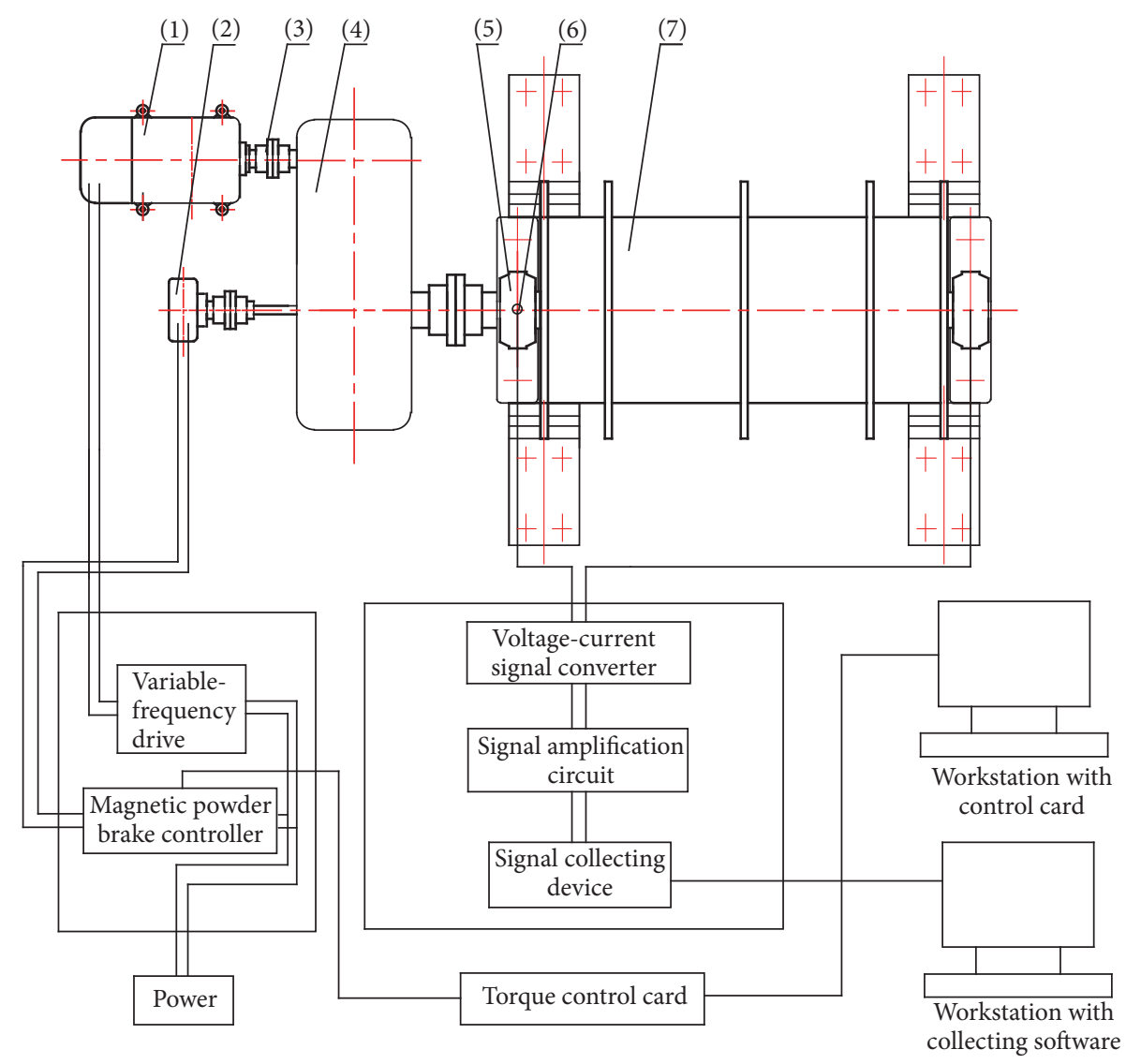

(a)

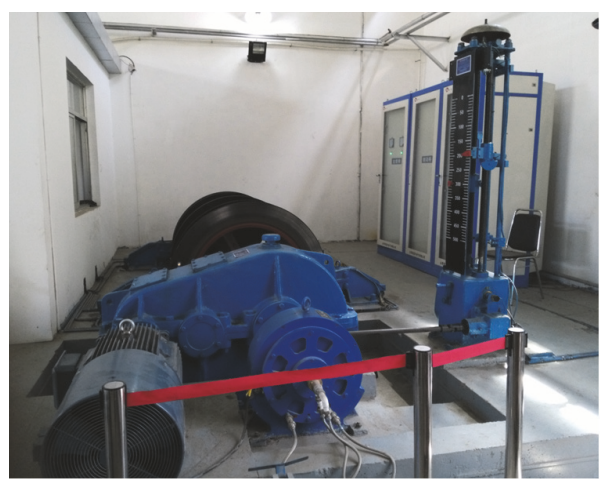

(b)

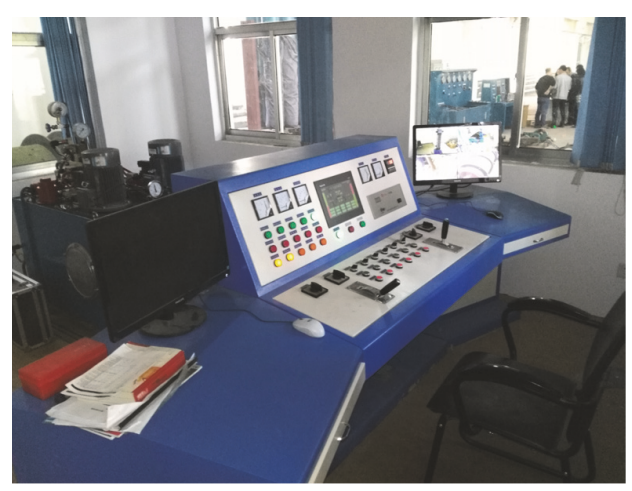

(c)

FIGURE 4: Load-recognition test bench for a low-speed rotor system. (a) Design schematic: (1) motor; (2) magnetic powder brake; (3) coupling; (4) reducer; (5) bearing; (6) displacement sensor; (7) low-speed rotor. (b) Recognition test bench. (c) Central control room.

rotor system. Concurrently, the vibration signals for the left rotor bearing were acquired in the radial horizontal direction. For example, when an impact torque of $M=1600 \mathrm{Nm}$ was applied for $0.5 \mathrm{~s}$, the corresponding displacement vibration signals were collected and the time domain waveform shows that the vibration signal changes over time (Figure 5). All other loads were tested in the same way.

By inputting the original vibration signals into the network directly, the signal features would be less prominent and therefore take more time to be recognized. To improve feature recognition, the original signal can be decomposed from high to low frequencies by EEMD. It is beneficial to the subsequent energy extraction of feature signals.

As an example, an impact load of $M=1600 \mathrm{Nm}$ was applied. Within the EEMD process, the selected Nstd was 0.02 , and $\mathrm{Ne}$ was 100. Accordingly, a 13th-order IMF and residual component were obtained. The effect of the EEMD decomposition of the vibration signals under this impact load is shown in Figure 5, demonstrating a front 6th-order IMF. After EEMD, the signal feature components in different time 

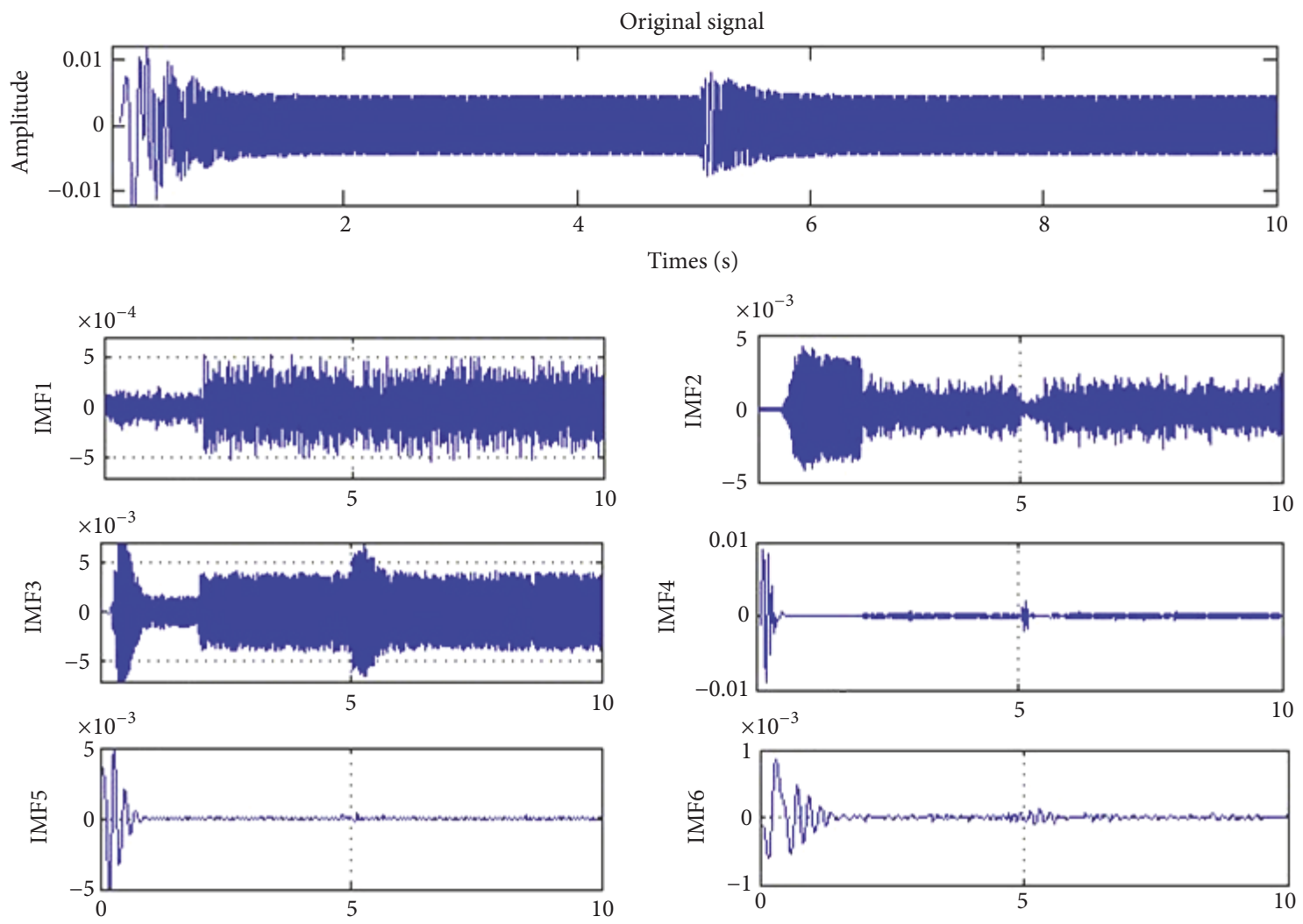

Figure 5: Ensemble empirical mode decomposition.

scales were included in IMF1 IMF6. The IMFs have different frequency components and bandwidths after EEMD, which change with the decomposed signal; therefore, EEMD is regarded as an adaptive band-pass filter. The vibration signals corresponding to other load types were processed similarly.

4.2. Energy Calculation of IMFs. Displacement signals are implemented by EEMD and, for each signal, the energy distribution of the IMF components is related to a certain applied load. As such, the energy of each signal component can be extracted as a feature [26]. Five load types were randomly selected, namely, impact load $(1600 \mathrm{Nm})$ for $0.5 \mathrm{~s}$, steady load $(1600 \mathrm{Nm})$, linear load $(t+1600 \mathrm{Nm})$, harmonic load $(100 \sin 4 \pi t+1550 \mathrm{Nm})$, and transient load $(1600 \mathrm{Nm})$ for $3 \mathrm{~s}$. There were 10 energy nodes gained after the energy calculation. In addition, the changes in energy due to the vibration signals were obtained. Energy changes can be represented by the node energy distribution (Figure 6).

In Figure 6, the horizontal axis represents the node sequence, while the vertical axis shows the energy value. Each line represents a change in energy with node order. The energy spectrum can directly reflect the different load types that correspond to different energy values as the energy values have a unique fluctuation pattern for each load type. In terms of IMF components within the same order, different energy values correspond with the vibration signals under different load conditions. As a consequence, the energy feature extraction can be applied to IMF components. In this way, it benefits the following load recognition.

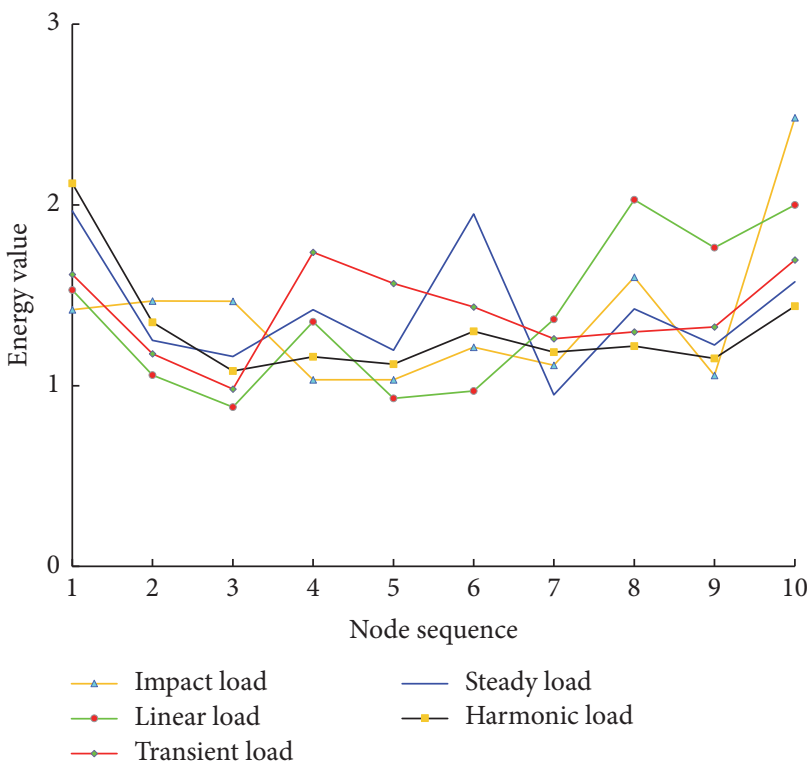

Figure 6: Energy distribution of nodes.

4.3. Load Qualitative Recognition. Applying the ANN method for load recognition, the input and output data usually include most of the dynamic characteristics describing an engineering structure. The trained ANN model can therefore store nonlinear mapping relationships between 
TABLE 3: Training sample data for load recognition in BP neural network.

\begin{tabular}{lcccccccccc}
\hline Type & $T_{1}$ & $T_{2}$ & $T_{3}$ & $T_{4}$ & $T_{5}$ & $T_{6}$ & $T_{7}$ & $T_{8}$ & $T_{9}$ & $T_{10}$ \\
\hline Impact (0.5 s) & 0.1023 & 0.1057 & 0.1056 & 0.0744 & 0.0744 & 0.0873 & 0.0802 & 0.1152 & 0.0761 & 0.1788 \\
Steady & 0.1393 & 0.0885 & 0.0822 & 0.1006 & 0.0847 & 0.1382 & 0.0673 & 0.1009 & 0.0868 & 0.1115 \\
Linear & 0.1102 & 0.0763 & 0.0634 & 0.0974 & 0.0670 & 0.0699 & 0.0985 & 0.1462 & 0.1271 & 0.1440 \\
Harmonic & 0.1615 & 0.1029 & 0.0824 & 0.0884 & 0.0853 & 0.0991 & 0.0903 & 0.0928 & 0.0876 & 0.1097 \\
Transient (3s) & 0.1146 & 0.0835 & 0.0696 & 0.1234 & 0.1111 & 0.1018 & 0.0895 & 0.0921 & 0.0941 & 0.1203 \\
\hline
\end{tabular}

TABLE 4: Testing sample data for load recognition in BP neural network.

\begin{tabular}{lcccccccccc}
\hline Number & $T_{1}$ & $T_{2}$ & $T_{3}$ & $T_{4}$ & $T_{5}$ & $T_{6}$ & $T_{7}$ & $T_{8}$ & $T_{9}$ & $T_{10}$ \\
\hline$(1)$ & 0.0313 & 0.0536 & 0.0535 & 0.1685 & 0.2233 & 0.2616 & 0.1324 & 0.0272 & 0.0097 & 0.0388 \\
$(2)$ & 0.0156 & 0.0041 & 0.0035 & 0.0051 & 0.0266 & 0.0211 & 0.1613 & 0.3205 & 0.3518 & 0.0905 \\
$(3)$ & 0.1484 & 0.0030 & 0.0011 & 0.0072 & 0.0670 & 0.7096 & 0.0339 & 0.0108 & 0.0057 & 0.0133 \\
\hline
\end{tabular}

vibration responses and applied loads. It also saves weights among neurons. And it can be used to accurately determine loads during operations.

The BPNN with three layers is a general function approximator, and it was chosen and applied in this study [30]. The corresponding vibration signals were measured in the experiment (Table 2). All the sample data were obtained after using EEMD and energy feature extraction for the vibration signals. Vibration signals corresponding to the loads to be recognized are the test samples, while the rest of the data are the training samples. Types of torque loads are outputs. Part of the training sample data is listed in Table 3, while Table 4 lists part of testing sample data.

For each signal, the number of characteristics was selected as 10 after EEMD. Moreover, there were five types of loads to be recognized. Therefore, for the designed BPNN, the number of input layer nodes was 10, and that of output layer nodes was 5 . The learning speed was found to be 0.01 , the inertia coefficient by the error was 0.2 , training time is 5000 , and training error was $1 e-3$. In accordance with the training error, the network was able to adjust the weights and threshold values during training.

The weights and threshold values were conserved after network training and the BPNN was capable of remembering and forecasting. The established network can screen the testing data. Finally, load recognition can be achieved.

The forecasting results using the BPNN classifier are shown in Figure 7. The abscissa shows the testing data samples, and the ordinate expresses category labels corresponding to the five load types, namely, impact load, steady load, linear load, harmonic load, and transient load. The "pink dots" show the actual categories of load, whereas the "blue stars" are the forecast categories. It shows intuitively that there is a good match between actual and forecasting values.

Based on the contrast between the recognized and real load types, the total recognition rate of the five load types was $92.38 \%$. It means that error is permitted. Load categories in the low-speed rotor system were well recognized by the BPNN.

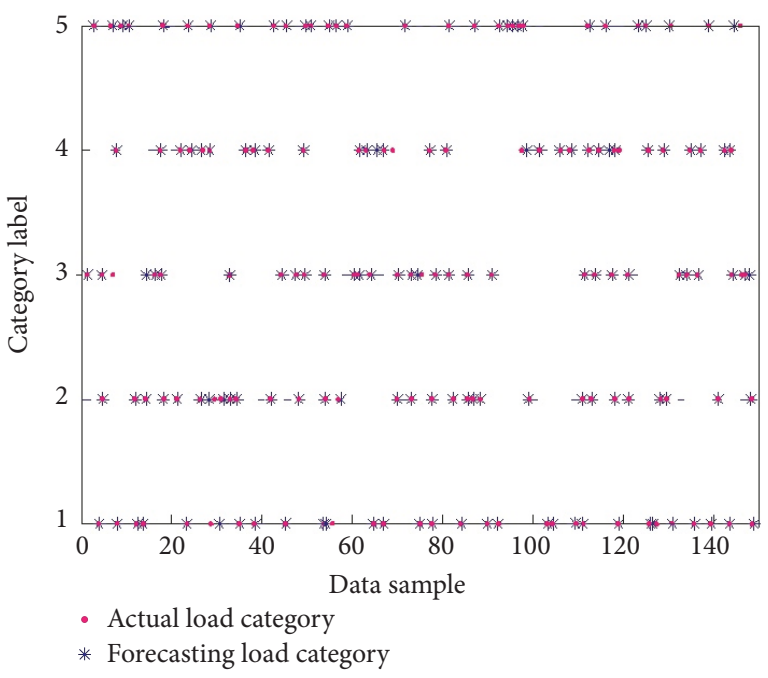

Figure 7: Prediction effect of BP network.

\section{Conclusions}

EEMD was presented to analyze different vibration signals, for a low-speed rotor system. In this way, the signal components of different scales were automatically distributed to the appropriate reference scales. Adding white noise to the signal before repeatedly using the EMD method eliminates mode mixing, which results in a clear decomposition of the signal.

Accurate data was obtained for the load-type recognition, after the energy feature extraction. Energy feature in each load type has a different pattern, which clearly distinguishes the load characteristics, thus benefitting load qualitative recognition.

The presented method is composed of EEMD, energy feature extraction, and BPNN, and it is based on the vibration signals from a low-speed rotor system. Comparing the experimental results with known values, a recognition rate of $92.38 \%$ was achieved and demonstrates that the presented 
recognition method is reliable and effective. The intended practical applications are low-speed rotor systems, such as large-scale cranes, as well as transportation, hoist, and heading equipment. Finally, the method could have potential uses in monitoring, control, fault diagnosis, and equipment management.

\section{Conflicts of Interest}

The authors declare that there are no conflicts of interest regarding the publication of this paper.

\section{Acknowledgments}

This work is supported by the National Natural Science Foundation of China (no. 51475318).

\section{References}

[1] S.-H. Wang, W. Guo, X.-Y. Xu, Y.-F. Liu, and W.-Y. Li, "Modeling unbalanced rotor system with continuous viscoelastic shaft by frequency-dependent shape function," Journal of Central South University, vol. 20, no. 12, pp. 3421-3430, 2013.

[2] S. W. Du, "A new method for fault diagnosis of mine hoist based on manifold learning and genetic algorithm optimized support vector machine," Elektronika Ir Elektrotechnika, vol. 123, no. 7, pp. 99-102, 2012.

[3] Z. C. Yang and Y. Jia, "The recognition of dynamic loads," Advances in Mechanics, vol. 45, no. 2, p. 30, 2015.

[4] B. J. Dobson and E. Rider, "A review of the indirect calculation of excitation forces from measured structural response data," Proceedings of the Institution of Mechanical Engineers, Part C: Journal of Mechanical Engineering Science, vol. 204, no. 2, pp. 69-75, 1990.

[5] H. Inoue, J. J. Harrigan, and S. R. Reid, "Review of inverse analysis for indirect measurement of impact force," Applied Mechanics Reviews, vol. 54, no. 6, pp. 503-524, 2001.

[6] Ł. Jankowski, "Off-line identification of dynamic loads," Structural and Multidisciplinary Optimization, vol. 37, no. 6, pp. 609623, 2009.

[7] M. Klinkov and C.-P. Fritzen, "An updated comparison of the force reconstruction methods," Key Engineering Materials, vol. 347, pp. 461-466, 2007.

[8] P. Czop, K. Mendrok, and T. Uhl, "Application of inverse linear parametric models in the identification of rail track irregularities," Archive of Applied Mechanics, vol. 81, no. 11, pp. 1541-1554, 2011.

[9] L. Yu and T. H. T. Chan, "Recent research on identification of moving loads on bridges," Journal of Sound and Vibration, vol. 305, no. 1-2, pp. 3-21, 2007.

[10] S. E. S. Karlsson, "Identification of external structural loads from measured harmonic responses," Journal of Sound and Vibration, vol. 196, no. 1, pp. 59-74, 1996.

[11] C.-Y. Fu, D.-S. Shan, and Q. Li, "Damage location recognition of railway bridge based on vibration response caused by vehicles," Journal of Modern Transportation, vol. 46, no. 5, pp. 719-725, 2011.

[12] L.-C. Zhang, H. Zhang, and X.-J. Zhang, "Continuous shearer load identification and properties research based on frequency domain method," Coal Mine Machinery, vol. 32, no. 6, pp. 77-79, 2011.
[13] C.-H. Huang, "An inverse non-linear force vibration problem of estimating the external forces in a damped system with timedependent system parameters," Journal of Sound and Vibration, vol. 242, no. 5, pp. 749-765, 2001.

[14] Y.-L. Zhang, G. Lin, Y. X. Wang, Z. Li, and G. Li, "An improved method of dynamic load recognition in time domain," Chinese Journal of Computational Mechanics, vol. 21, no. 2, pp. 209-215, 2004.

[15] J.-S. Wang, Research on structural dynamic load identification based on modal analysis method [Ph.D. dissertations], Dalian University of Technology, 2013.

[16] Y. Y. Hu, Z. J. Shuai, W. Y. Li, and Z. G. Liu, "Status quo of study on machine's load recognition technique," Noise and Vibration Control, vol. 31, no. 4, pp. 1-5, 2011.

[17] Y. Z. Feng, C. X. Fan, and Y. Zhang, "The progress of dynamic load recognition," Journal of Shangqiu Teachers College, vol. 24, no. 9, p. 59, 2008.

[18] P. Zhou, Q. Zhang, Z. J. Shuai, and W. Li, "Review of research and development status of dynamic load recognition in time domain," Noise and Vibration Control, vol. 34, no. 1, p. 10, 2014.

[19] G. Yan and L. Zhou, "Impact load identification of composite structure using genetic algorithms," Journal of Sound and Vibration, vol. 319, no. 3-5, pp. 869-884, 2009.

[20] R. Hashemi and M. H. Kargarnovin, "Vibration base recognition of impact force using genetic algorithm," International Journal of Mechanical Systems Science and Engineering, vol. 26, no. 1-2, pp. 204-210, 2007.

[21] M. E. Williams and M. I. Hoit, "Bridge pier live load analysis using neural networks," Advances in Engineering Software, vol. 35, no. 10-11, pp. 645-652, 2004.

[22] J. H. Lin, X. L. Guo, H. Zhi, W. P. Howson, and F. W. Williams, "Computer simulation of structural random loading identification," Computers \& Structures, vol. 79, no. 4, pp. 375387, 2001.

[23] K. K. Tahboub, M. Barghash, M. Arafeh, and O. Ghazal, "An ANN-GA framework for optimal engine modeling," Mathematical Problems in Engineering, vol. 2016, Article ID 6180758, 9 pages, 2016.

[24] X. M. Wang, Neural Network Introduction, Science Press, Beijing, China, 2017.

[25] K. Zhang and Z.-J. Yang, "Identification of load categories in rotor system based on vibration analysis," Sensors, vol. 17, no. 7, pp. 1-16, 2017.

[26] Z. H. Wu and N. E. Huang, "Ensemble empirical mode decomposition: a noise-assisted data analysis method," Advances in Adaptive Data Analysis, vol. 1, no. 1, pp. 1-41, 2009.

[27] Y. G. Lei, Z. J. He, and Y. Y. Zi, "Application of the EEMD method to rotor fault diagnosis of rotating machinery," Mechanical Systems and Signal Processing, vol. 23, no. 4, pp. 1327-1338, 2009.

[28] Y.-G. Chen and Z.-Y. Zhong, "Modal parameter identification of a cable-stayed bridge based on CEEMD and DATA-SSI algorithm," Journal of Vibration and Shock, vol. 35, no. 8, pp. 166-172, 2016.

[29] Y. Liu, Research on monitoring methods of tool condition based on ensemble empirical mode decomposition and support vector machine [Ph.D. dissertations], Southwest Jiaotong University, 2012.

[30] G. Zhao, H. Wang, G. Liu, and Z. Q. Wang, "Optimization of stripping voltammetric sensor by a back propagation artificial neural network for the accurate determination of $\mathrm{Pb}$ (II) in the presence of Cd(II)," Sensors, vol. 16, no. 9, article 1540, p. 3, 2016. 


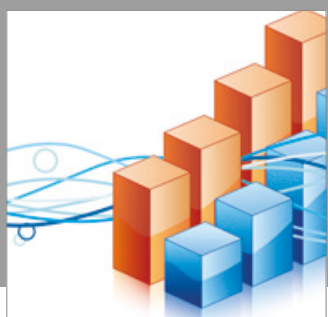

Advances in

Operations Research

vatersals

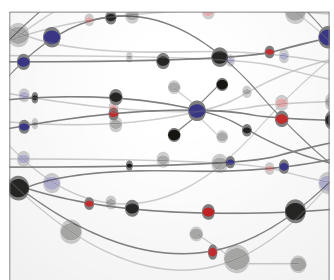

\section{The Scientific} World Journal
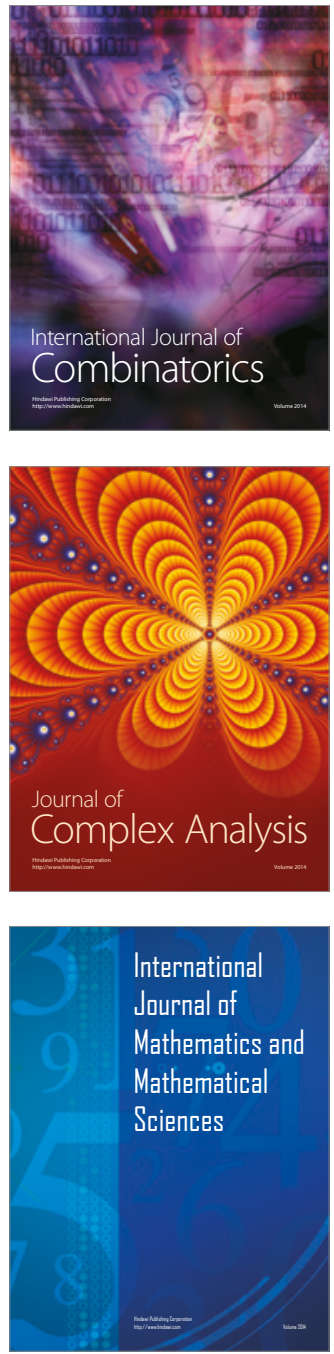
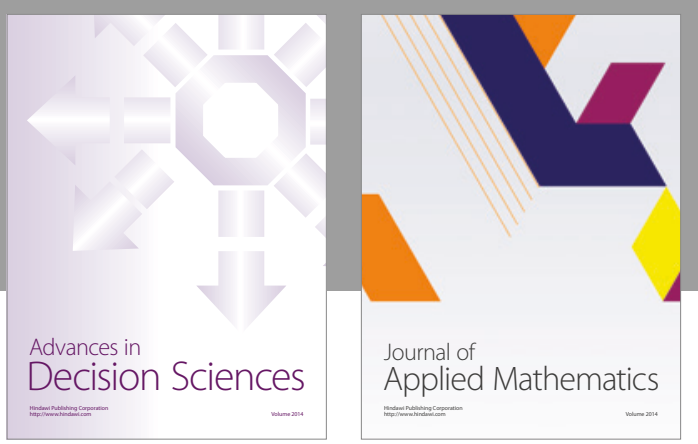

Algebra

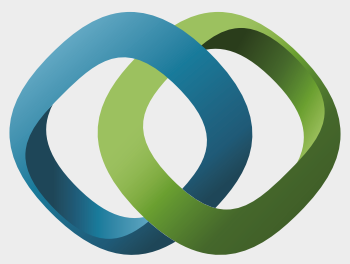

\section{Hindawi}

Submit your manuscripts at

https://www.hindawi.com
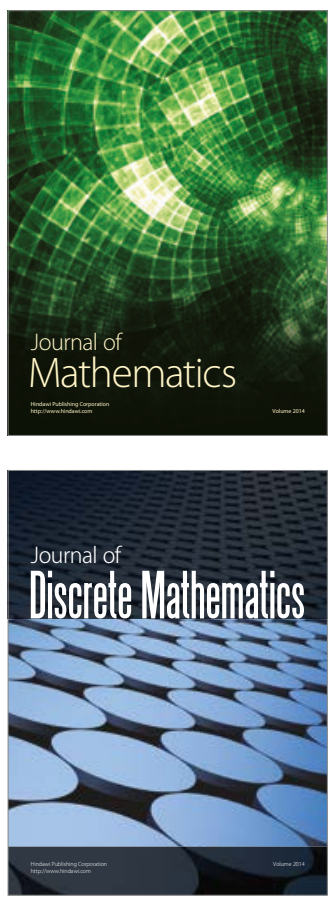

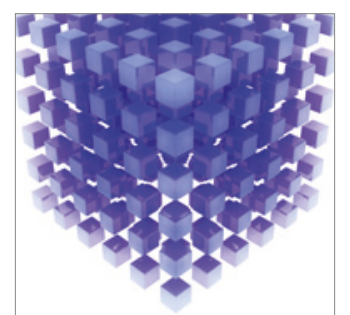

Mathematical Problems in Engineering
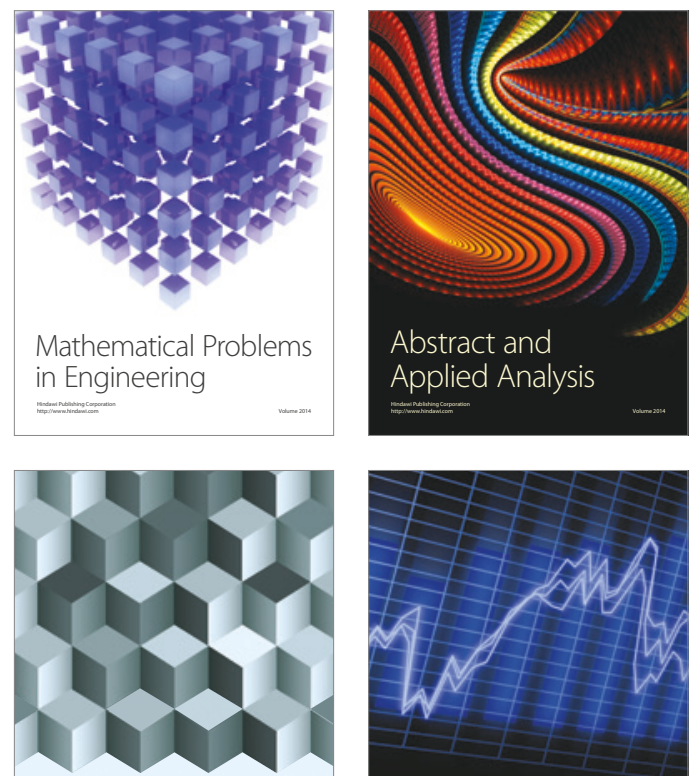

Journal of

Function Spaces

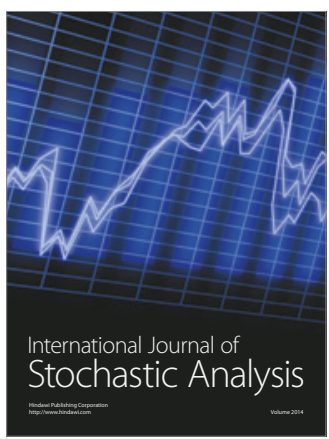

Probability and Statistics
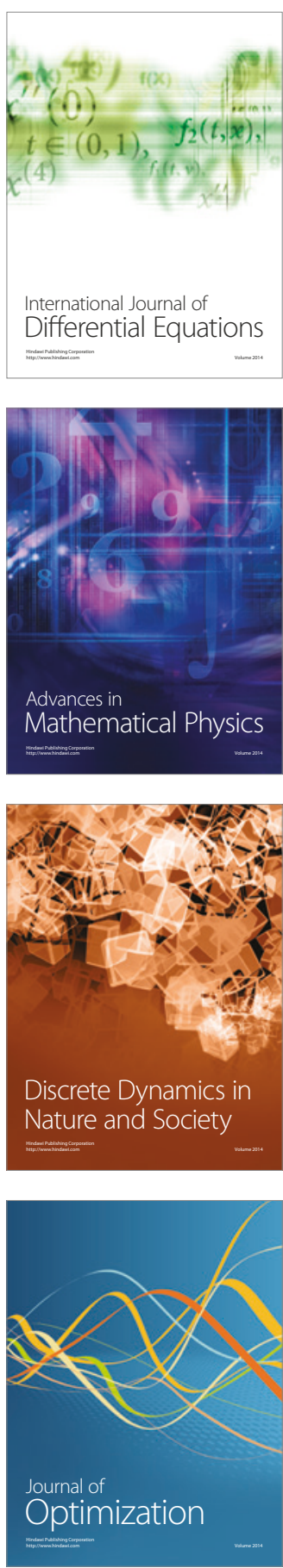